\section{Orofacial mechanisms and swallowing abilities in a geriatric population in India}

Shenbagavalli Mahalingam, MASLP, KV Vijay Kumar, MASLP

\begin{abstract}
Background. Presbyaphagia is common among elderly people. This cross-sectional study aimed to compare different geriatric age-groups in South India in terms of orofacial mechanisms, swallowing abilities, and dietary patterns.
\end{abstract}

Methods: Residents from three care homes for the elderly in Tamil $\mathrm{Nadu}$, South India who were $\geq 60$ years and conscious and oriented during assessment were recruited between January 2016 and December 2016. Participants were classified into age-groups of 60-70 years, 7180 years, and $>80$ years. A protocol was developed and verified by two speech language pathologists. The protocol included orofacial mechanism examination, swallowing assessment, and dietary pattern assessment.

Results: A total of 67 men and 57 women aged 60 to 92 years were classified into age-groups of $60-70$ years $(n=45), 71-80$ years $(n=44)$, or $>80$ years $(n=35) .69 .4 \%$ of participants were taking medications for hypertension, diabetes, dyslipidaemia, or any of the combinations. Older age-groups showed more drooping of facial appearance $(\mathrm{p}<0.001)$, habitual posture of lips $(\mathrm{p}<0.001)$, tongue appearance $(p<0.001)$, dentures $(p<0.001)$, and lip mobility functions such as pursing $(p<0.001)$, puckering $(p<0.001)$, tongue movements and mobility ( $p=0.003)$, jaw mobility $(p<0.001)$, dental alignment $(p=0.001)$, and occlusion $(\mathrm{p}=0.001)$. Older age-groups also showed more tongue placement $(p<0.001)$, oral spillage $(p<0.001)$, drooling/increased secretions $(p=0.005)$, pocketing of food $(p<0.001)$, presence of cough during swallowing $(p<0.001)$, reduced strength of cough $(p<0.001)$, and presence of wet/gurgling voice immediately after swallowing $(\mathrm{p}<0.001)$. None had nasal regurgitation. As age increased, feeding time increased $(\mathrm{p}<0.001)$ and preferred food consistency changed from solids to semisolids and then to purees $(\mathrm{p}<0.001)$.

Conclusions: Older age-groups have poorer orofacial mechanisms and swallowing abilities. Elderly people in India are less concerned about swallowing issues because Indian food has a wide variety of food consistencies.

Key words: Deglutition disorders; Sarcopenia

\section{ORIGINAL ARTICLE}

Department of Speech, Language and Hearing Sciences, Sri Ramachandra Institute of Higher Education and Research
Correspondence to: KV Vijay Kumar. Email: vijaykumarkv@yahoo.in 


\section{INTRODUCTION}

Presbyphagia is the second most commonly reported symptom in geriatric medicine. ${ }^{1-3}$ Sarcopenic changes in the oropharyngeal muscles result in reduced intake, ${ }^{4,5}$ prolonged chewing and/or reduced lingual pressure, ${ }^{1,6}$ reduced ability to control food or liquids in the mouth, ${ }^{7}$ and slowed swallowing. ${ }^{8}$ In addition, alteration in central drive for feeding, changes in taste, smell, and chemosensory inputs lead to weak cough and reduced intake of food. ${ }^{8}$ Increased frequency of aspiration and pneumonia is attributed to the biomechanical changes in swallowing structures. ${ }^{1}$ Presbyphagia affects the quality of life of self and caregivers. ${ }^{9}$ Safe swallowing of food with different consistencies and textures ensures to meet the nutritional demand in elderly. ${ }^{10,11}$ Recent research in geriatrics emphasises on dietary modification and intake of supplementary sources. ${ }^{12}$

In India, life expectancy of the age-group of $\geq 60$ years has increased $17.5 \%$ to $19.1 \% .^{13}$ Indian diet can be modified in texture and consistency without altering the nutritional value. This study aimed to compare different geriatric age-groups in South India in terms of orofacial mechanisms, swallowing abilities, and dietary patterns.

\section{METHOD}

This study was approved by the ethics committee of our institution (Ref: IEC-NI/15/APR/46/15). Informed consent was obtained from each participant. Between January 2016 and December 2016, residents were recruited from three care homes for the elderly in Tamil Nadu, South India who were aged $\geq 60$ years and conscious and oriented during assessment. Those with known neurological conditions, cognitive impairment, head and neck surgeries, or chronic obstructive pulmonary disease were excluded. Mini Mental State Examination was administered to rule out the presence of neurocognitive disturbances.

Participants were classified into age-groups of 60-70 years, 71-80 years, and $>80$ years. A protocol was developed and verified by two speech language pathologists who had $\geq 10$ years of experience in management of swallowing disorders. The protocol included orofacial mechanism examination (of appearance and functions of structures at rest and during swallowing), swallowing assessment, and dietary pattern assessment (TABLE $\mathbf{1}$ ). Different consistencies of staple food viz liquids, semisolids, and solids were used.

TABLE 1

Parameters of orofacial mechanisms and swallowing abilities

\begin{tabular}{ll}
\hline Parameters & Assessment \\
\hline Swallowing with teeth apart & $\begin{array}{l}\text { During swallowing, the lower jaw drops down, the upper and lower teeth separate and provide } \\
\text { room for the tongue thrust to a more comfortable position. }\end{array}$ \\
$\begin{array}{l}\text { Protrusion of tongue while swallowing } \\
\text { During swallowing, the tongue protrudes to the anterior incisors. } \\
\text { Oral expectoration/spillage }\end{array}$ & $\begin{array}{l}\text { During food chewing, a part of the bolus spills out owing to inadequate lip seal. } \\
\text { Nasal regurgitation }\end{array}$ \\
$\begin{array}{l}\text { Drooling/increased secretions during swallowing, the bolus is regurgitated through nose owing to velopharyngeal inadequacy. } \\
\text { swallowing }\end{array}$ & $\begin{array}{l}\text { During swallowing, spillage of saliva anteriorly is referred as drooling. } \\
\text { Pocketing of food, mouth odour }\end{array}$ \\
$\begin{array}{l}\text { Coughing/choking/throat clearing } \\
\text { while swallowing }\end{array}$ & $\begin{array}{l}\text { After swallowing, food lodging in lateral vestibule is referred as pocketing of food. } \\
\text { before consuming the next spoon of food. }\end{array}$ \\
$\begin{array}{l}\text { Gurgling/wet voice after swallowing } \\
\text { Strength of cough }\end{array}$ & $\begin{array}{l}\text { Immediately after swallowing, a change in voice quality results in a wet gurgling voice. } \\
\text { determines the strength of the cough. }\end{array}$ \\
$\begin{array}{l}\text { Prolonged feeding time } \\
\text { Dehydration, temperature spike, } \\
\text { pneumonia }\end{array}$ & $\begin{array}{l}\text { Taken from in-house medical records } \\
\text { Weight loss }\end{array}$ \\
$\begin{array}{l}\text { Food preference } \\
\text { Taken from the annual in-house medical records }\end{array}$ \\
\hline $\begin{array}{l}\text { After consumption of different consistencies of food, participants were asked about preferred } \\
\text { food consistency. }\end{array}$
\end{tabular}


TABLE 2

Comparison of three age-groups in terms of orofacial mechanisms and swallowing abilities

\begin{tabular}{|c|c|c|c|c|}
\hline \multirow[t]{2}{*}{ Parameter } & \multicolumn{3}{|c|}{ Age-group } & \multirow[t]{2}{*}{$\mathrm{p}$ Value } \\
\hline & $\begin{array}{c}60-70 \\
\text { years } \\
(n=45)^{\star}\end{array}$ & $\begin{array}{c}71-80 \\
\text { years } \\
(n=44)^{\star}\end{array}$ & $\begin{array}{l}>80 \text { years } \\
(n=35)^{\star}\end{array}$ & \\
\hline Age, y & $64.9 \pm 3.3$ & $75.6 \pm 3.4$ & $85.3 \pm 3.3$ & \\
\hline No. of men:women & $27: 18$ & $17: 27$ & $23: 12$ & \\
\hline \multicolumn{5}{|l|}{ Orofacial mechanisms } \\
\hline Facial appearance & & & & $<0.001$ \\
\hline Normal & $44(97.8)$ & $15(34.1)$ & $3(8.6)$ & \\
\hline Drooping & $1(2.2)$ & $29(65.9)$ & $32(91.4)$ & \\
\hline \multicolumn{5}{|l|}{ Lips appearance } \\
\hline Normal & $45(100)$ & $44(100)$ & $35(100)$ & - \\
\hline Deviant & 0 & 0 & 0 & \\
\hline Lips habitual posture & & & & $<0.001$ \\
\hline Closed & $45(100)$ & $43(97.7)$ & $19(54.3)$ & \\
\hline Parted & 0 & $1(2.3)$ & $16(45.7)$ & \\
\hline \multicolumn{5}{|l|}{ Lips mobility } \\
\hline Pursing & & & & $<0.001$ \\
\hline Adequate & $45(100)$ & $44(100)$ & $22(62.9)$ & \\
\hline Inadequate & 0 & 0 & $13(37.1)$ & \\
\hline Pouting & & & & $<0.001$ \\
\hline Absent & $43(95.6)$ & $18(40.9)$ & $3(8.6)$ & \\
\hline Present & $2(4.4)$ & $26(59.1)$ & $32(91.4)$ & \\
\hline Spreading & & & & 0.278 \\
\hline Adequate & $45(100)$ & $44(100)$ & $34(97.1)$ & \\
\hline Inadequate & 0 & 0 & $1(2.9)$ & \\
\hline Jaw mobility & & & & $<0.001$ \\
\hline Drooping & 0 & 0 & $1(2.9)$ & \\
\hline Excessive & 0 & 0 & $1(2.9)$ & \\
\hline Insufficient & 0 & $14(31.8)$ & $25(71.4)$ & \\
\hline Sufficient & $45(100)$ & $30(68.2)$ & $8(22.9)$ & \\
\hline Tongue appearance at rest & & & & $<0.001$ \\
\hline Atrophy & 0 & $7(15.9)$ & $17(48.6)$ & \\
\hline Atrophy, tremors & 0 & 0 & $4(11.4)$ & \\
\hline Normal & $44(97.8)$ & $35(79.5)$ & 7 (20.0) & \\
\hline Tremors & $1(2.2)$ & $2(4.5)$ & $7(20.0)$ & \\
\hline Tongue protrusion & & & & 0.003 \\
\hline Normal & $45(100)$ & 40 (90.9) & $27(77.1)$ & \\
\hline Tremors & 0 & $44(9.1)$ & 35 (22.9) & \\
\hline Tongue mobility & & & & $<0.001$ \\
\hline Adequate & $42(93.3)$ & $41(93.2)$ & 17 (48.6) & \\
\hline Absence of elevation & $3(6.7)$ & 0 & 0 & \\
\hline $\begin{array}{l}\text { Absence of lateral } \\
\text { movement }\end{array}$ & 0 & $1(2.3)$ & $1(2.9)$ & \\
\hline Inability to lick their lips & 0 & 0 & 5 (14.3) & \\
\hline $\begin{array}{l}\text { Inability to sweep from } \\
\text { alveolar ridge to palate }\end{array}$ & 0 & $2(4.5)$ & $12(34.2)$ & \\
\hline Teeth & & & & $<0.001$ \\
\hline Dentures & 0 & $9(20.5)$ & $11(31.4)$ & \\
\hline Missing & $10(22.2)$ & $23(52.3)$ & $20(57.1)$ & \\
\hline Carries & $10(22.2)$ & $32(77.7)$ & 31 (88.8) & \\
\hline Tooth alignment & & & & 0.001 \\
\hline Misaligned & 0 & 0 & $1(2.9)$ & \\
\hline Spaced & $1(2.9)$ & $2(4.5)$ & $4(11.4)$ & \\
\hline Normal & $44(97.8)$ & $33(75)$ & 19 (54.3) & \\
\hline Not applicable & 0 & $9(20.5)$ & $11(31.4)$ & \\
\hline $\begin{array}{l}\text { Tooth condition (excluding } \\
\text { those with dentures) }\end{array}$ & & & & $<0.001$ \\
\hline Normal & $27(60.0)$ & 14 (27.3) & $6(17.1)$ & \\
\hline Decayed & $18(40.0)$ & 23 (52.3) & $18(51.4)$ & \\
\hline
\end{tabular}

TABLE 2 (cont'd)

\begin{tabular}{lcccc}
\hline Parameter & \multicolumn{3}{c}{ Age-group } & p Value \\
\cline { 2 - 4 } & $\begin{array}{c}60-70 \\
\text { years } \\
(\mathrm{n}=45)^{\star}\end{array}$ & $\begin{array}{c}71-80 \\
\text { years } \\
(\mathrm{n}=44)^{\star}\end{array}$ & $\begin{array}{c}>80 \text { years } \\
(\mathrm{n}=35)^{\star}\end{array}$ & \\
& $42(93.3)$ & $31(70.5)$ & $18(51.4)$ & 0.001 \\
\hline Tooth occlusion & 0 & $9(20.5)$ & $11(31.4)$ & \\
$\quad$ Normal & $3(6.7)$ & $4(9.1)$ & $6(17.1)$ & \\
$\quad$ Not applicable & & & & \\
$\quad$ Over jet & $45(100)$ & $44(100)$ & $35(100)$ & \\
Hard palate appearance & & & & \\
$\quad$ Normal & $45(100)$ & $44(100)$ & $35(100)$ & \\
Soft palate & $45(100)$ & $44(100)$ & $35(100)$ & \\
$\quad$ Normal appearance & $45(100)$ & $44(100)$ & $34(97.1)$ & 0.278 \\
$\quad$ Symmetry in mobility & $45(100)$ & $44(100)$ & $35(100)$ & \\
$\quad$ Presence of gag reflex & 45 & & & \\
$\quad$ Normal uvula & 45 & & &
\end{tabular}

Swallowing abilities

Swallowing with teeth apart Present Absent

Protrusion of tongue while swallowing Present Absent

Oral expectoration/spillage Present Absent

Nasal regurgitation Present Absent

Drooling/increased secretions during swallowing Present Absent

Pocketing of food, mouth odour

Present

Absent

Coughing/choking/throat clearing while swallowing Present Absent

Gurgling/wet voice after swallowing Present Absent

Strength of cough Adequate Weak

Prolonged feeding time Present Absent

Dehydration, temperature spike, pneumonia

Present

Absent

Weight loss

Present Absent

Food preference

No preference

Solids

Solids + semisolids

Semisolids

Semisolids + purees

Purees

0

$1(2.3) \quad 3(8.6)$

$45(100) \quad 43(97.7) \quad 32(91.4)$

0.089

$0 \quad 1(2.3) \quad 15(42.9)$

$45(100) \quad 43(97.7) \quad 20(57.1)$

$0 \quad 5(11.3) \quad 20(57.1)$

$45(100) \quad 39(88.6) \quad 15(42.9)$

$<0.001$

$45(100) \quad 44(100) \quad 35(100)$

0.005

$0 \quad 0 \quad 4(11.4)$

$45(100) \quad 44(100) \quad 31(88.6)$

5 (11.1) $17(38.6) \quad 20(57.1)$

$40(88.9) \quad 27(61.4) \quad 15(42.9)$

$<0.001$

$1(2.2) \quad 18(40.9) \quad 26(74.3)$

$44(97.8) \quad 26(59.1) \quad 9(25.7)$

$<0.001$

$4(8.9) \quad 18(40.9) \quad 24(68.6)$

41 (91.1) $26(59.1) \quad 11(31.4)$

$44(97.8) \quad 43(97.7) \quad 22(62.9)$

$1(2.2) \quad 1(2.3) \quad 13(37.1)$

$1(2.2) \quad 18(40.9) \quad 25(71.4)$ $<0.001$

$44(97.8) \quad 26(59.1) \quad 10(28.6)$

0.075

$\begin{array}{lll}0 & 0 & 2(5.7)\end{array}$

$45(100) \quad 44(100) \quad 33(94.3)$

$1(2.2) \quad 10(22.7) \quad 9(25.7)$

0.006

$44(97.8) \quad 34(77.3) \quad 26(74.3)$

$\begin{array}{cll}22(48.9) & 0 & 2(5.7) \\ 6(13.3) & 3(6.8) & 0 \\ 0 & 1(2.3) & 1(2.9) \\ 17(37.8) & 35(79.6) & 7(20.0) \\ 0 & 2(4.6) & 8(22.9) \\ 0 & 3(6.8) & 16(45.7) \\ 0 & 0 & 1(2.9)\end{array}$ $<0.001$ Liquids

* Data are presented as mean \pm standard deviation or No. (\%) of participants 
Statistical analysis was performed using SPSS (Macintosh version 25; IBM Corp, Armonk [NY], US). The three age-groups were compared using Chi-squared test.

\section{RESULTS}

A total of 67 men and 57 women aged 60 to 92 (mean \pm standard deviation, $74 \pm 8.89$ ) years were classified into age-groups of 60-70 years $(n=45), 71-80$ years $(n=44)$, or $>80$ years $(n=35)$ [TABLE 2]. $69.4 \%$ of participants were taking medications for hypertension $(15.3 \%)$, diabetes $(21.6 \%)$, dyslipidaemia $(0.02 \%)$, or any of the combinations (24.3\%).

The three age-groups were comparable in terms of lips spreading and gag reflex $(\mathrm{p}=0.278)$, but older age-groups showed more drooping of facial appearance $(\mathrm{p}<0.001)$, habitual posture of lips $(p<0.001)$, tongue appearance $(p<0.001)$, dentures $(\mathrm{p}<0.001)$, and lip mobility functions such as pursing $(p<0.001)$, puckering $(p<0.001)$, tongue movements and mobility $(\mathrm{p}=0.003)$, jaw mobility $(\mathrm{p}<0.001)$, dental alignment $(\mathrm{p}=0.001)$, and occlusion $(\mathrm{p}=0.001)$.

Older age-groups also showed more tongue placement $(\mathrm{p}<0.001)$, oral spillage $(\mathrm{p}<0.001)$, drooling/increased secretions $(\mathrm{p}=0.005)$, pocketing of food $(\mathrm{p}<0.001)$, presence of cough during swallowing $(p<0.001)$, reduced strength of cough $(p<0.001)$, and presence of wet/gurgling voice immediately after swallowing $(\mathrm{p}<0.001)$. None had nasal regurgitation.

As age increased, feeding time increased $(\mathrm{p}<0.001)$ and preferred food consistency changed from solids to semisolids and then to purees $(\mathrm{p}<0.001)$. In the age-group of $60-70$ years, $48.9 \%(n=22)$ did not have preference for any particular consistency/texture and $51 / 1 \%$ preferred semisolids or solids. In the agegroup of $71-80$ years, $91 \%(n=40)$ preferred solids, semisolids, and/or purees. In the age-group of $>80$ years, $68.6 \%$ preferred purees and/or semisolids and $20.0 \%$ preferred semisolids only. The three agegroups were comparable in terms of swallowing with teeth apart, presence of dehydration, temperature hike, and history of pneumonia.

\section{DISCUSSION}

\section{Oral stage}

Atrophy of the skin and loss of collagen and elastin fibres results in weak facial muscle tone and elasticity and hence drooping of perioral musculature. Poor lip function and jaw instability were observed in participants aged $>80$ years, consistent with other studies that have reported age-related decline in endurance, mobility, and strength in elderly people resulting in poor lip closure and jaw instability. ${ }^{14,15}$ The incidences of atrophy of the tongue and tremors in the tongue increase with age. Decreased tongue muscle fibre diameter may contribute to tongue atrophy. ${ }^{6}$ Lower endurance may induce tremors in the tongue. Decreased skeletal muscle fibre diameter results in reduced tongue strength and range of motion of the tongue and hence weak propulsion of bolus to the posterior of the oral cavity. ${ }^{16}$

Tongue thrust is common in elderly people. ${ }^{17} \mathrm{In}$ participants aged $>80$ years, protrusion of tongue was observed in edentulous patients and those with missing teeth or dentures. Oral spillage, drooling, and pocketing of food was observed more frequently in older age-groups. Proprioception, temperature sensitivity, and oral stereognosis decline as age increases. ${ }^{8}$ This may result in altered sensation in oral cavity and lip, jaw, and tongue mobility, leading to increased secretions during swallowing, oral spillage, and pocketing of food. No structural and functional deviances were observed in hard palate, soft palate, and uvula.

\section{Pharyngeal stage}

The primary concerns in the pharyngeal phase of swallowing are weak coughing and gurgling voice immediately after swallowing. ${ }^{18}$ The reason for weak cough is multifactorial, but it is hypothesised that changes in bronchial smooth muscle tone may result in an altered cough reflex. ${ }^{19}$ Delay in laryngeal vestibule closure also results in penetration/aspiration in elderly people. ${ }^{20}$ Gurgling voice immediately after swallowing may indicate presence of swallowing disorder. Delayed trigger in swallowing reflex resulting in premature swallowing and reduced pharyngeal peristalsis may lead to penetration of bolus and gurgling voice. ${ }^{1}$ These findings suggest that elderly people are at risk of aspiration, but only two participants aged $>80$ years reported to have pneumonia recently, and most participants did not develop any complication secondary to swallowing issues.

Sarcopenic changes in the oral structures, pharyngeal, and upper oesophageal sphincter limit the choice over food consistency and texture. ${ }^{21}$ 
Purees and semisolids are preferred as age increases. Liquids (thin and thick) increase the risk of aspiration, and solids are difficult to swallow owing to poor dentition. ${ }^{12}$ Altering the food consistency to meet the nutritional demands and needs indicate the coping/ compensatory mechanism for swallowing issues.

Traditional Indian food has a wide variety of consistencies. Rice can be consumed as mashed steamed rice, upma (soft solid), porridge (thick liquid consistency), or murukku (solid). Anecdotally, diversity in staple food and choice over food enables elderly people to opt for comfortable consistencies. Although $83.3 \%$ of participants had preferences for food, none reported of change in diet pattern as a supplementary process. Rather, they reported it as a variant of a regular diet, and swallowing difficulties may go unnoticed. In swallowing rehabilitation, combination of compensatory manoeuvres and modification in food consistency and texture improves swallowing functions. ${ }^{22-24}$ Use of isolated modified food consistencies in the management of swallowing difficulty is questionable.

\section{Limitations}

Our swallowing assessment was subjective. Objective evaluation using the modified barium swallow could have validated the subjective assessment of swallowing parameters. Analysing the effects of medication on feeding may yield interesting results. Weight loss, food preference, dehydration, and pneumonia should have been documented for a longitudinal study.

\section{CONCLUSION}

Older age-groups have poorer orofacial mechanisms and swallowing abilities. Elderly people in India are less concerned about swallowing issues because Indian food has a wide variety of food consistencies.

\section{DECLARATION}

No financial support was obtained to carry out this research. The authors have no conflict of interest to disclose.

\section{REFERENCES}

1. Logemann JA, Pauloski BR, Rademaker AW, Colangelo LA, Kahrilas PJ, Smith CH.Temporal and biomechanical characteristics of oropharyngeal swallow in younger and older Men. J Speech
Lang Hear Res 2000;43:1264-74. Crossref

2. Rumeau P, Vellas B. Dysphagia, a geriatric point of view [in French]. Rev Laryngol Otol Rhinol (Bord) 2003;124:331-3.

3. McCullough GH, Wertz RT, Rosenbek JC, Suiter D, McCoy SC. Defining swallowing function by age: promises and pitfalls of pigeonholing. J Speech Lang Hear Res 2007;48:1280-93. Crossref

4. Salva A, Coll-Planas L, Bruce S, et al. Nutritional assessment of residents in long-term care facilities (LTCFs): recommendations of the task force on nutrition and ageing of the IAGG European region and the IANA. J Nutr Health Aging 2009;13:475-83. Crossref

5. Bourdel-Marchasson I. How to improve nutritional support in geriatric institutions. J Am Med Dir Assoc 2010;11:13-20. Crossref

6. Ortega O, Cabre M, Clave P. Oropharyngeal dysphagia: aetiology and effects of ageing. J Gastroenterol Hepatol Res 2014;3:1049-54.

7. Yoshikawa M, Yoshida M, Nagasaki T, Tanimoto K, Tsuga K, Akagawa $Y$. Influence of aging and denture use on liquid swallowing in healthy dentulous and edentulous older people. J Am Geriatr Soc 2006;54:444-9. Crossref

8. Morley JE. Decreased food intake with aging. J Gerontol A Biol Sci Med Sci 2001;56:81-8. Crossref

9. Lieu PK, Chong MS, Seshadri R. The impact of swallowing disorders in the elderly. Ann Acad Med Singapore 2001;30:148-54.

10. Atherton M, Bellis-Smith N, Cichero J, Suter M. Texture-modified foods and thickened fluids as used for individuals with dysphagia: Australian standardised labels and definitions. Nutr Diet 2006;64(Suppl 2). Crossref

11. British Dietetic Association. National Descriptions for Texture Modification in Adults. Available from: http://www.bda.uk.com/ publications/statements/NationalDescriptorsTextureModification Adults.pdf. Accessed 27 June 2010.

12. Sura L, Madhavan A, Carnaby G, Crary MA. Dysphagia in the elderly: management and nutritional considerations. Clin Interv Aging 2012;7:287-98. Crossref

13. Elderly in India. Available from: http://mospi.nic.in/sites/default/ files/publication_reports/ElderlyinIndia_2016.pdf Accessed 1 November 2018.

14. Dinse HR, Kleibel N, Kalisch T, Ragert F, Wilimzig C, Tegenthoff $\mathrm{M}$. Tactile coactivation resets age-related decline of human tactile discrimination. Ann Neurol 2006;60:88-94. Crossref

15. Nicosia MA, Hind JA, Roecker EB, et al. Age effects on the temporal evolution of isometric and swallowing pressure. J Gerontol A Biol Sci Med Sci 2000;55:M634-40. Crossref

16. Fukunaga A, Uematsu $H$, Sugimoto $K$. Influences of aging on taste perception and oral somatic sensation. J Gerontol A Biol Sci Med Sci 2005;60:109-13. Crossref

17. Rofes L, Arreola V, Romea M, et al. Pathophysiology of oropharyngeal dysphagia in the frail elderly. J Neurogastroenterol Motil 2010;22:851-8. Crossref

18. Dejaeger M, Liesenborghs C, Dejaeger. Presbyphagia. Sem Dysphagia. Available from: https://www.intechopen.com/books/ seminars-in-dysphagia/presbyphagia.

19. Lalley PM. The aging respiratory system: pulmonary structure, function and neural control. Respir Physiol Neurobiol 2013;187:199-210. Crossref

20. Sue Eisenstadt E. Dysphagia and aspiration pneumonia in older adults. J Am Acad Nurse Pract 2010;22:17-22. Crossref

21. Ney DM, Weiss JM, Kind AJ, Robbins J. Senescent swallowing: impact, strategies, and interventions. Nutr Clin Pract 2009;24:395413. Crossref

22. Luk JK, Chan DK. Preventing aspiration pneumonia in older people: do we have the'know-how'? Hong Kong Med J 2014;20:421-7. Crossref

23. Baijens LW, Clavé P, Cras P, et al. European Society for Swallowing Disorders - European Union Geriatric Medicine Society white paper: oropharyngeal dysphagia as a geriatric syndrome. Clin Interv Aging 2016;11:1403-28. Crossref

24. Speyer R, Baijens L, Heijnen M, Zwijnenberg I. Effects of therapy in oropharyngeal dysphagia by speech and language therapists: a systematic review. Dysphagia 2010;25:40-65. Crossref 